\title{
MATURAÇÃO DE FRUTOS DE Caesalpinia echinata Lam., PAU-BRASIL ${ }^{1}$
}

Francismar Francisco Alves Aguiar ${ }^{2}$, Marcos Mecca Pinto ${ }^{2}$, Armando Reis Tavares $^{2}$ e Shoey Kanashiro ${ }^{2}$

RESUMO - Estudaram-se parâmetros indicativos da maturação de frutos de árvores de Caesalpinia echinata, visando determinar a melhor época de colheita para propagação. Foram utilizadas 10 plantas-matriz cultivadas em Mogi-Guaçu, SP, nas quais foram etiquetadas 250 inflorescências no pico da floração para acompanhamento da maturação. As coletas de frutos tiveram início a partir da $5^{a}$ semana após a antese, prolongando-se até a $9^{a}$, com intervalos de sete dias. Em cada coleta, analisaram-se os parâmetros: comprimento, largura e teor de água dos frutos; teor de água das sementes (após três dias de exposição ao sol para deiscência do fruto e extração da semente); porcentagem de germinação e peso de matéria seca de frutos, sementes e plântulas. Observou-se, ainda, a coloração dos frutos como parâmetro visual de maturação das sementes. O experimento foi repetido por três anos. Os testes de germinação foram realizados em caixas Gerbox contendo vermiculita umedecida com água destilada. As sementes foram colocadas para germinar em câmara regulada para $30^{\circ} \mathrm{C}$ e fotoperíodo de 12 horas $\mathrm{O}$ delineamento estatístico utilizado foi inteiramente casualizado com quatro repetições de 25 sementes por parcela, nos anos de 1991, 1992 e 1993. As leituras de germinação foram realizadas nos $4^{\circ}$ e $8^{\circ}$ dias após a semeadura. Pelos resultados, conclui-se que o momento ideal para coleta dos frutos de C. echinata é no estádio de pré-dispersão (entre a $8^{\circ}$ e $9^{\circ}$ após a antese) visualizado através da coloração, quando estes mudam de verde para castanho.

Palavras-chave: Caesalpinia echinata, pau-brasil, germinação de sementes e maturação de sementes.

\section{MATURATION OF FRUITS OF Caesalpinia echinata Lam. (BRAZIL WOOD)}

\begin{abstract}
Several indicative parameters regarding fruit maturity of Caesalpinia echinata were studied in order to establish the ideal harvest time. Ten matrix plants were cultivated in Moji-Guaçu, $S P\left(22^{\circ} 11^{\prime}\right.$ to $1022^{\circ} 18^{\prime} S$ and $47^{\circ} 13^{\prime}$ to $47^{\circ} 20^{\prime} \mathrm{WG}$ ), altitude between $600 \mathrm{~m}$ and $730 \mathrm{~m}$. The climate of the region is classified as Cwa. Two hundred and fifty blossoms were tagged during the the peak blooming period for maturation analysis. Fruit-harvesting schedule ranged from the fifth to the ninth week after anthesis, with seven-day intervals. The following parameters where analyzed for each harvest: fruit length and width; fruit moisture content; seed moisture content (after natural pre-drying); germination percentage; seed, fruit and seedling dry matter weight. Fruit colors were also observed and used as a visual parameter of seed maturation. The experiment was repeated for three years. The germination tests were performed in Gerbox boxes, with vermiculite watered with distilled water. The seeds were set to germinate in B.O.D cameras, at $30^{\circ} \mathrm{C}$, with a 12-hour photoperiod. The experimental design was completely randomized with four replications of 25 seeds per plot in 1991, 1992 and 1993. The germination was analyzed on the 4th and 8th days after seeding. The results showed that the ideal harvest time for Brazil-wood fruits is at the pre-dispersion stage, which can be identified visually through colors, when they change from green to chestnut-brown.
\end{abstract}

Keywords: Caesalpinia echinata Lam., Brazil wood, germination and maturity of seeds.

\footnotetext{
${ }^{1}$ Recebido em 22.04.2005 e aceito para publicação em 13.09.2006.

${ }^{2}$ Pesquisador Científico do Instituto de Botânica, Caixa Postal 4005, 01061-970 São Paulo-SP. E-mail:<atavares2005@ yahoo.com.br>.
} 


\section{INTRODUÇÃOO}

Caesalpinia echinata Lam. é uma árvore pertencente à família Caesalpiniaceae (CRONQUIST, 1981)e, conforme a Lei 6.607 de 07/12/1978 (BRASIL, 1978), foi instituída a árvore nacional. A espécie está ameaçada ou em perigo de extinção, sendo conhecida no Brasil como pau-brasil. A planta apresenta grande potencial ornamental graças às suas características morfológicas de grande beleza (SOUZA, 1982). Sua madeira foi intensamente explorada durante quase quatro séculos para fabricação de tinta de escrever e, principalmente, para tingir tecido, sendo atualmente utilizada na arborização urbana e a confecção de arcos de violinos (AGUIAR e PINHO, 1996). A espécie pode ser encontrada na costa atlântica brasileira desde o Rio Grande do Norte até o Rio de Janeiro (AGUIAR e AOKI, 1983), não tolera geada e tem área de distribuição natural em regiões com até $200 \mathrm{~m}$ de altitude, muito embora tenha sido encontrada em Vitória da Conquista, BA, em altitudes de 550 a 600 m (CARVALHO, 1994). É uma espécie semi-heliófila, classificada como clímax de acordo com os critérios de Budowski (1965).

Existem poucos estudos científicos sobre a maturação de frutos e sementes de C. echinata (RAMALHO, 1978; AGUIAR e BARBOSA, 1985; AGUIAR, 1990; 1997; 2001, BORGES et al., 2003). Porém, há vários estudos sobre maturação de sementes de espécies nativas, como: Inga uruguenses (FIGLIOLIA e KAGEYAMA, 1994), Acácia polyphyla DC (ARAÚJO NETO, 2001), Miconia cinnamomifolia (DC.) Naud. (PEREIRA e MANTOVANI, 2001) e Cedrela fissilis Vell. (CORVELLO et al., 1999). No entanto, diversos autores, estudando tecnologia das sementes, concluíram que os estádios de maturação fisiológica de máximo vigor e de máxima porcentagem de germinação são praticamente coincidentes e indicados como o ponto ideal para a colheita (CAMARGO e VECCHI, 1971; DELOUCHE e CARDWELL, 1960; JACINTHO e CARVALHO, 1974; POPINIGIS, 1985). Adeterminação de maturidade fisiológica dos frutos é de fundamental importância para orientar a época ideal de colheita, pois auxilia o planejamento dessa operação no processamento, na secagem, no armazenamento e no controle de qualidade. Dessa forma, a determinação da época adequada de colheita garante o máximo poder germinativo e vigor das sementes (CARVALHO e NAKAGAWA, 1988). Teoricamente, o ponto de maturidade fisiológica seria o ponto ideal de colheita.
O objetivo deste trabalho foi estudar a maturação de frutos de C. echinata e definir parâmetros indicativos de maturação com a finalidade de determinar a época mais adequada para a colheita dos frutos com semente de alta qualidade em relação à germinação e ao vigor.

\section{MATERIAL E MÉTODOS}

O estudo utilizou 10 plantas-matrizes de $C$. echinata, cultivadas em arboreto experimental, pertencente à reserva Biológica e Estação Experimental de Mogi-Guaçu, SP (22 $2^{\circ} 11^{\prime}$ a $22^{\circ} 18^{\prime}$ 'S e $47^{\circ} 13^{\prime}$ a $47^{\circ} 20^{\prime} \mathrm{W}$ ). A altitude média da área varia de 600 a 730 m. O clima da região é do tipo Cwa, com precipitação média anual de $1.386 \mathrm{~mm}$ e temperatura média anual de $20,75^{\circ} \mathrm{C}$, mínima de $14,69^{\circ} \mathrm{C}$ e máxima de 28,88 ${ }^{\circ} \mathrm{C}$, com umidade relativa do ar (UR) de 76\% (AGUIAR, 1992). Durante três anos $(1991,1992,1993)$ foram etiquetadas 250 inflorescências (10 inflorescências/ árvore/ano) na antese, no mês de outubro, para acompanhamento da maturação dos frutos (novembro/ dezembro). As coletas foram iniciadas a partir da quinta semana após a antese, prosseguindo até a nona, com intervalos de sete dias. Nas coletas foram analisados os parâmetros: coloração, separada em três categorias (verde, parcialmente castanho e castanho), comprimento, largura, peso da matéria seca e teor de água dos frutos (100 frutos por coleta); peso de matéria seca e teor de água das sementes (após três dias de exposição ao sol para deiscência do fruto e extração da semente); e porcentagem de germinação e peso de matéria seca das plântulas (coletadas no oitavo dia após a semeadura - 16 plântulas por coleta).

Os testes de germinação foram realizados em caixas plásticas transparentes (Gerbox) contendo vermiculita de granulação média, umedecida com água destilada. As sementes foram colocadas para germinar em Câmara Fanem B.O.D. e regulada para $30^{\circ} \mathrm{C} \pm \mathrm{e}$ $1{ }^{\circ} \mathrm{C}$ e fotoperíodo de 12 horas. As leituras de germinação foram realizadas no quanto e oitavo dias após a semeadura (AGUIAR et al., 1996.), sendo consideradas sementes germinadas aquelas com protusão de raiz primária de no mínimo $2 \mathrm{~mm}$ e as sementes que desenvolveram plântulas normais. O teor de água e peso de matéria seca dos frutos e das sementes, assim como o peso de matéria seca das plântulas, 
foram determinado pelo método da estufa $\left(105^{\circ} \mathrm{C}\right.$ por 24 horas), conforme Brasil (1992). As análises estatísticas quanto à porcentagem de germinação foram realizadas com os dados transformados em arco-seno $(\% / 100)^{1 / 2}$. O índice de velocidade de emergência (IVE) foi calculado pela metodologia proposta por Popiningis (1985). O delineamento estatístico utilizado foi inteiramente casualizado com quatro repetições de 25 sementes por parcela, nos anos de 1991, 1992 e 1993.

\section{RESULTADOS E DISCUSSÃO}

De acordo com os dados do Quadro 1, a porcentagem de germinação das sementes e o IVE de C. echinata foram semelhantes nos três anos de experimentação, exceto na quinta semana de 1992, quando foi menor. Nesse quadro, os resultados, em razão da semana de coleta, evidenciaram que na quinta semana após a antese a geminação foi significativamente menor que as demais. Apesar de não haver diferenças significativas entre a germinação de sementes colhidas após a sexta semana, houve uma tendência de diminuição da porcentagem de germinação na nona semana após a antese.

Valores altos de germinação obtidos nessas semanas também foram constatados por Aguiar (1990). O IVE também refletiu essa tendência, em que os menores índices se referem à $5^{\mathrm{a}}$ semana, quando a porcentagem de germinação e os acréscimos mais significativos no vigor, expresso pelo índice de velocidade de emergência, ocorreram a partir da sexta semana. Os resultados foram semelhantes aos obtidos por Brasil (1992), estudando a maturação fisiológica de sementes de cedro (Cedrela fissilis), tendo esse autor sugerido que a germinação e o vigor das sementes são respostas ao acúmulo de matéria seca que podem se manifestar determinado tempo depois de atingido o máximo peso de matéria seca de sementes.

Ainda nessas semanas, verifica-se, no Quadro 2 , que o peso de matéria seca dos frutos, as sementes e as plântulas foram superiores em relação à quinta semana. Quando a semente atinge o máximo de peso de matéria seca, de acordo com Carvalho e Nakagawa (1988), ela alcança o ponto de maturação fisiológica, ou seja, encontra-se no máximo de sua viabilidade, e a deterioração é mínima. O conteúdo de matéria seca tem sido apontado como um bom índice de maturação, e o seu valor máximo geralmente coincide com a máxima capacidade de germinação.

Os teores de água no fruto e na semente foram menores na nona semana após a antese (Quadro 2). Portanto, os dados de germinação do Quadro 1, combinados com os dados do Quadro 2, indicam que entre a sexta e nona semanas após a antese as sementes encontravam-se em boas condições de germinação, porém foi na nona semana que as sementes estavam com o máximo de matéria seca acumulada, menor teor de água e, portanto, na maturidade fisiológica, conforme indicado em Popinigs (1985).

Quadro 1 - Maturação de sementes de Caesalpinia echinata Lam. (pau-brasil): média de germinação (\%) de sementes e índice de velocidade de emergência (IVE) em função do ano de coleta dos frutos e do tempo decorrido da antese até a coleta

Table 1 - Seed maturation of Caesalpinia echinata Lam. (Brazil-wood): seed germination average (\%) and the germination velocity index (IVE) according the year of fruit harvest and elapsed time between anthesis and harvest

\begin{tabular}{|c|c|c|c|c|c|c|}
\hline \multirow{3}{*}{$\begin{array}{c}\text { Semanas após } \\
\text { a Antese }\end{array}$} & \multicolumn{6}{|c|}{ Ano de Coleta dos Frutos } \\
\hline & \multicolumn{2}{|c|}{1991} & \multicolumn{2}{|c|}{1992} & \multicolumn{2}{|c|}{1993} \\
\hline & Germinação (\%) & IVE & Germinação (\%) & IVE & Germinação (\%) & IVE \\
\hline $5^{\mathrm{a}}$ & 47 c B & $1,00 \mathrm{c} \mathrm{B}$ & 28 b B & 0,68 b B & 58 b B & $1,40 \mathrm{~b} \mathrm{~B}$ \\
\hline $6^{\mathrm{a}}$ & 95 a b A & 2,13 a $\mathrm{A}$ & 97 a $\mathrm{A}$ & 2,42 a $\mathrm{A}$ & 90 a $\mathrm{A}$ & 2,15 a $\mathrm{A}$ \\
\hline $7^{\text {a }}$ & $98 \mathrm{ab} \mathrm{A}$ & $1,30 \mathrm{bc} \mathrm{B}$ & 92 a $\mathrm{A}$ & 2,14 a $\mathrm{A}$ & 94 a $\mathrm{A}$ & 1,93 a $\mathrm{AB}$ \\
\hline $8^{\text {a }}$ & 100 a $\mathrm{A}$ & $1,38 \mathrm{bc} \mathrm{AB}$ & 97 a $\mathrm{A}$ & 2,42 a $\mathrm{A}$ & 90 a $\mathrm{A}$ & 2,23 a $\mathrm{A}$ \\
\hline $9^{a}$ & $80 \mathrm{~b} \mathrm{~A}$ & $1,73 \mathrm{ab} \mathrm{AB}$ & 92 a $\mathrm{A}$ & 2,20 a $\mathrm{A}$ & 86 a $\mathrm{A}$ & 1,95 a $\mathrm{AB}$ \\
\hline C.V. & 12,18 & 22,78 & 9,77 & 11,27 & 12,89 & 13,63 \\
\hline
\end{tabular}

Médias seguidas pela mesma letra maiúscula e minúscula na mesma coluna não diferem entre si, em níveis de 1 e $5 \%$, respectivamente, pelo teste de Tukey. 
Quadro 2 - Maturação de frutos de Caesalpinia echinata Lam. (pau-brasil): médias e intervalos de confiança do peso de matéria seca de frutos, sementes e plântulas, teor de água (\%) de frutos e sementes obtidos entre os anos de 1991,1992 e 1993 e da $5^{\text {a à }} 9^{\text {a }}$ semana após a antese

Table 2 - Caesalpinia echinata Lam. (Brazil-wood) fruit maturation: means and confidence interval for fruit, seed and seedling dry matter weight and fruit and seed water content (\%), harvested between the 5th and 9 th week after anthesis, during 1991, 1992 and 1993

\begin{tabular}{|c|c|c|c|c|c|}
\hline \multirow{2}{*}{$\begin{array}{c}\text { Semanas } \\
\text { após antese }\end{array}$} & \multicolumn{3}{|c|}{ Peso de matéria seca (mg.unidade ${ }^{-1}$ ) } & \multicolumn{2}{|c|}{ Teor de água (\%) } \\
\hline & Fruto & Semente & Plântulas & Fruto & Semente \\
\hline $5^{a}$ & $140 \pm 37$ & $10 \pm 01$ & $14 \pm 01$ & $54,5 \pm 12,55$ & $12,2 \pm 0,18$ \\
\hline $6^{a}$ & $175 \pm 31$ & $24 \pm 05$ & $18 \pm 08$ & $45,6 \pm 04,51$ & $15,0 \pm 0,12$ \\
\hline $7^{a}$ & $185 \pm 33$ & $21 \pm 07$ & $23 \pm 08$ & $44,7 \pm 11,13$ & $14,7 \pm 4,25$ \\
\hline $8^{a}$ & $171 \pm 32$ & $24 \pm 03$ & $18 \pm 03$ & $47,4 \pm 02,13$ & $18,2 \pm 6,58$ \\
\hline $9^{a}$ & $177 \pm 18$ & $28 \pm 03$ & $22 \pm 05$ & $33,9 \pm 16,15$ & $11,4 \pm 2,03$ \\
\hline
\end{tabular}

Observações de campo indicam que após a nona semana, ocorre a dispersão natural das sementes (AGUIAR, 2001). Neste estudo, na nona semana os frutos apresentavam coloração castanha (Quadro 3). Essa alteração da cor de frutos é coincidente com a maturidade fisiológica das sementes (POPINIGS, 1985; CARVALHOeNAKAGAWA, 1988) e tem sido observado em diversas espécies nativas do Brasil, conforme Pinã-Rodrigues e Aguiar (1993) em Cordia goeldiana Haber (freijó), Anadenanthera macrocarpa (Benth.) Brenan e Miroxylum Balsamum (L.) Harm. (cabreúva). Corvello et al. (1999) obtiveram os mesmos resultados em frutos de Cedrela Fissilis Vell. (cedro), em que entre a $25^{\mathrm{a}}$ e a $31^{\mathrm{a}}$ semana após a antese os frutos sofreram alteração na coloração, passando de completamente verde para marrom-esverdeada, o que evidencia a diminuição e posterior cessamento da translocação de fotossintetizados, corroborando as afirmações de que a coloração dos frutos, associada a outros índices, é uma característica que pode ser utilizada como indicadora da maturidade fisiológica de sementes. Segundo Barbedo et al. (2002), que estudaram a influência da coloração de sementes de C. echinata, a despeito de diferenças na coloração externa e no conteúdo de água essas não apresentaram diferenças significativas quanto à matéria seca, dimensões das plântulas germinadas e porcentagem de germinação das sementes.

A melhor fase para a colheita de frutos de C. echinata com alta qualidade de sementes foi aquela na qual se constataram elevada germinação e alto vigor associados a baixos teores de água nos frutos e nas sementes. Essa fase correspondeu a nona semana após a antese, quando os frutos estavam iniciando a abertura dos frutos e a deiscência natural das sementes. A extração de sementes de frutos colhidos nessa fase foi mais rápida quando estes expostos ao sol e cobertos com tela para evitar a dispersão das sementes. Nas fases anteriores, os teores de água dos frutos foram mais altos (Quadro 2), sendo necessário um tempo maior de exposição ao sol para abertura dos frutos e extração das sementes, mas evita-se a perda de sementes pela dispersão natural, considerando que o fruto da espécie apresenta deiscência explosiva. Esses dados confirmam as observações fenológicas realizadas por Aguiar (2001). De acordo com o Quadro 3, entre a quinta e a nona semana após a antese as dimensões dos frutos (comprimento e largura) foram semelhantes.

Quadro 3 - Maturação de frutos de Caesalpinia echinata Lam. (pau-brasil): cor dos frutos, médias e intervalos de confiança de comprimento e largura dos frutos, obtidos durante os anos de 1991, 1992 e 1993 e da $5^{\text {a }}$ a 9 a semanas após a antese

Table 3 - Caesalpinia echinata Lam. (Brazil-wood) fruit maturation: fruit color, average e error bands from fruit length and width, harvested between the 5th and 9th week after anthesis during 1991, 1992 and 1993

\begin{tabular}{cccc}
\hline Semanas & & Características dos frutos \\
\cline { 2 - 5 } após a antese & Cor & Comprimento $(\mathrm{cm})$ & Largura $(\mathrm{cm})$ \\
\hline $5^{\mathrm{a}}$ & verde & $7,31 \pm 0,40$ & $2,54 \pm 0,20$ \\
$6^{\mathrm{a}}$ & parcial castanho & $6,98 \pm 0,13$ & 2,60 \\
$7^{\mathrm{a}}$ & parcial castanho & 6,11 & $2,48 \pm 0,03$ \\
$8^{\text {a }}$ & parcial castanho & $6,95 \pm 0,09$ & $2,29 \pm 0,14$ \\
$9^{\text {a }}$ & castanho & $6,92 \pm 0,35$ & $2,75 \pm 0,60$ \\
\hline & & $7,02 \pm 0,14$ & $2,53 \pm 0,10$ \\
\hline
\end{tabular}

R. Árvore, Viçosa-MG, v.31, n.1, p.1-6, 2007 


\section{CONCLUSÃO}

Nas condições do presente trabalho, foi possível concluir que:

- A colheita dos frutos de Caesalpinia echinata Lam. pode ser realizada entre a $6^{\mathrm{a}}$ e a $9^{\mathrm{a}}$ semana após a antese, quando as sementes apresentam o máximo de germinação.

- Para obtenção de sementes com alta qualidade fisiológica, o período mais adequado para a colheita é a fase de pré-dispersão, num intervalo de sete dias antes do ponto de maturidade fisiológica, entre a $8^{\text {a }}$ e a $9^{\mathrm{a}}$ semana após a antese, quando os frutos estão na fase de transição de coloração; passando de verde para castanho na época da colheita os frutos de Caesalpinia echinata Lam. estavam com 7,02 $( \pm 0,14)$ $\mathrm{cm}$ de comprimento e 2,53 $( \pm 0,1) \mathrm{cm}$ de largura.

\section{REFERÊNCIAS}

AGUIAR, F.F.A. Comportamento ecológico de Caesalpinia echinata Lam. (pau-brasil) cultivado em arboreto experimental. I. Revista Árvore, v.16, n.3, p.255-261, 1992.

AGUIAR, F.F.A. Observações preliminares sobre a fenologia de Caesalpinia echinata Lam. (Paubrasil) em arboreto experimental. In: CONGRESSO FLORESTAL BRASILEIRO, 6 , 1990, Campos do Jordão. Anais... Campos do Jordão: 1990. p.591-596.

AGUIAR, F.F.A. Maturação de sementes de Caesalpinia echinata Lam. - Pau-brasil. In: CONGRESSO NACIONAL DE BOTÂNICA, XLVIII, Resumos... Crato: 1997. p.44.

AGUIAR, F.F.A. Fenologia do pau-brasil (Caesalpinia echinata Lam.) em Mogi Guaçu, SP. Revista Ecossistema, v.26, n.1, p.107-112, 2001.

AGUIAR, F.F.A.; AOKI, K. Regiões de ocorrência de Pau-Brasil (Caesalpinia echinata Lam.). Silvicultura, v.28, p.1-5, 1983.

AGUIAR, F.F.A.; BARBOSA, J.M. Estudos de conservação de longevidade de sementes de paubrasil (Caesalpinia echinata Lam.). Revista Ecossistema, v.10, p.145-150, 1985.
AGUIAR, F.F.A.; PINHO, R.A. Pau-brasil (Caesalpinia echinata Lam.). 2. ed. São Paulo: Instituto de Botânica, 1996. 14p.

AGUIAR, F.F.A. et al. Influência do tamanho sobre a germinação de sementes de Caesalpinia echinata Lam (Pau-brasil), Revista Brasileira de Sementes, v.18, n.2, p.283-285, 1996.

ARAÚJO NETO, J.C. Aspectos fenológicos, caracterização, germinação e armazenamento de sementes de Acacia polyphylla (DC) Naud. 2001. 109 f. Tese (Doutorado em Agronomia) Universidade Estadual Paulista, Jaboticabal, 2001.

BARBEDO, C.J.; BILIA, D.A.C.; FIGUEIREDORIBEIRO, R.C.L. Tolerância à dessecação e armazenamento de sementes de Caesalpinia echinata Lam. (pau-brasil), espécie da Mata Atlântica. Revista Brasileira de

Botânica, v.25, n.4, p.431-440, 2002.

BORGES, I.F. et al. Maturação de sementes de Caesalpinia echinata Lam. (pau-brasil) em bosque implantado no estado de São Paulo. In: CONGRESSO DE ECOLOGIA DO BRASIL, 6., 2003, Fortaleza: Anais... Fortaleza, 2003. p.256-257.

BRASIL. Ministério da Agricultura e Reforma Agrária. Regras para Análise de Sementes. Brasília: SNDA/DNDV/CLAV, 1992.365p.

BUDOWSKI, A. Distribution of tropical rain forest species in the light of. successional progress. Turrialba, v. 15, p.40-42, 1965.

CAMARGO, C.P.A.; VECCHI, C. Pesquisa em tecnologia de sementes. Porto Alegre: ABRATES, 1971. 45p.

CARVALHO, P.E.R. Espécies florestais brasileiras: recomendações silviculturais, potencialidades e uso da madeira. Brasília: Embrapa, 1994. 640p.

CARVAlho, N.M.; NAKAGAWA, J. Sementes: ciência, tecnologia e produção. 3. ed. Campinas: Fundação Cargil, 1988. 424p.

CORVELLO, W.B.V. et al. Maturação fisiológica de sementes de cedro (Cedrela fissilis Vell.).

Revista Brasileira de Sementes, v.21, n.2, p.23-29, 1999.

R. Árvore, Viçosa-MG, v.31, n.1, p.1-6, 2007 
CRONQUIST, A. An integrated system of classification of flowering plants. New York: Columbia University Press, 1981. 1262p.

DELOUCHE, J.C.; CALDWELL, W.P. Seed vigor and vigor tests. Proceedings of the Association of Official Seed Analysts, v.50, p.124-129, 1960.

BRASIL. Leis e Decretos - Lei 6.607. Diário Oficial da República Federativa do Brasil, Brasília, 07 de dezembro, 1978.

FIGLIOLIA, M.B.; KAGEYAMA, P.Y. Maturação de sementes de Inga uruguensis Hook et Arn. em floresta riparia do rio Mogi Guaçu, município de Mogi Guaçu, SP. Revista do Instituto Florestal, v.6, p.13-52, 1994.

JACINTHO, J.B.C.; CARVALHO, N.M. Maturação de sementes de soja (Glycine max L. Merril).

Científica. Jaboticabal, v.1, n.1, p.81-87, 1974.
PEREIRA, S.T.; MANTOVANI, W. Maturação e dispersão de Miconia cinnamomifolia (DC) Naud. na Res. Biol. de Poço das Antas, município de Silva Jardim, RJ, Brasil. Acta Botanica Brasília, v.15, n.3, p.335-348, 2001.

PINÃ-RODRIGUES, F.C.M.; AGUIAR, I..B. Maturação e dispersão de sementes In: AGUIAR, I.R.; PINÃ-RODRIGUES, F.C.M; FIGLIOLIO, M.B.(Ed.) Sementes Florestais Tropicais. Brasília: ABRATES, 1993. p.215-74

POPININGIS, F. Fisiologia da semente. Brasília: AGIPLAN, 1985. 289p.

RAMALHO, R.S. Pau-brasil (Caesalpinia echinata Lam.). Viçosa, MG: Universidade Federal de Viçosa, 1978. 11p. (Boletim de extensão, 12).

SOUZA, H.M. O pau-brasil. O Estado de São Paulo, 10 de dezembro de 1982. (Suplemento Agrícola, 1386). 